\title{
DISCOVERY OF X-RAY PULSATIONS FROM THE NEXT GEMINGA, PSR J1836+5925
}

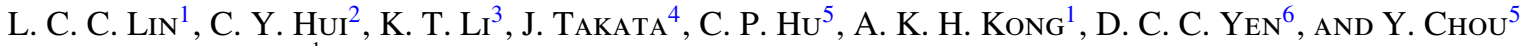 \\ ${ }^{1}$ Institute of Astronomy, National Tsing-Hua University, Hsinchu 30013, Taiwan \\ 2 Department of Astronomy and Space Science, Chungnam National University, Daejeon, South Korea; cyhui@ @nu.ac.kr \\ ${ }^{3}$ Department of Biological Science and Technology, China Medical University, Taichung 40402, Taiwan \\ ${ }^{4}$ Department of Physics, University of Hong Kong, Pokfulam Road, Hong Kong, China \\ ${ }^{5}$ Graduate Institute of Astronomy, National Central University, Jhongli 32001, Taiwan \\ ${ }^{6}$ Department of Mathematics, Fu Jen Catholic University, New Taipei City 24205, Taiwan \\ Received 2014 August 3; accepted 2014 August 19; published 2014 September 3
}

\begin{abstract}
We report recent XMM-Newton investigations of PSR J1836+5925, the "next Geminga," which determined an $\mathrm{X}$-ray pulsation of $\sim 173.3 \mathrm{~ms}$. Its X-ray periodicity is consistent with the $\gamma$-ray ephemeris at the same epoch. The $\mathrm{X}$-ray folded light curve has a sinusoidal structure that is different from the double-peaked $\gamma$-ray pulse profile. We have also analyzed the X-ray phase-averaged spectra which shows that the X-ray emission from PSR J1836+5925 is thermal dominant. This suggests that the $\mathrm{X}$-ray pulsation mainly originates from the modulated hot spot on the stellar surface.
\end{abstract}

Key words: gamma rays: general - pulsars: general - pulsars: individual (PSR J1836+5925) radiation mechanisms: non-thermal - radiation mechanisms: thermal - X-rays: general

Online-only material: color figure

\section{INTRODUCTION}

The launch of the Fermi Gamma-Ray Space Telescope in 2008 provides the opportunity to examine $\gamma$-rays emitted from compact objects, and $117 \gamma$-ray pulsars have been detected with the first $3 \mathrm{yr}$ of archival data (Abdo et al. 2013). Among all the pulsars detected by Fermi, 36 were directly found via a blind search in the $\gamma$-ray band and 34 of them were identified as radio-quiet with a threshold of $30 \mu \mathrm{Jy}$ in radio flux density at $1400 \mathrm{MHz}$. The population of radio-quiet $\gamma$-ray pulsars is expanding due to the continuous accumulation of Fermi data and the improvement of the searching schemes (e.g., weighting the $H$ test statistic (TS); Kerr 2011). This has imposed constraints on the pulsar emission geometry, which suggests that the radio emission and $\gamma$-rays are not produced in the same region of the pulsar magnetosphere. While the radio emission originates from the cones emerging near the stellar surface, the pulsed $\gamma$-rays are generally accepted to arise from the outer magnetosphere (see Cheng \& Zhang 1998; Takata et al. 2006, 2008). Radioquiet pulsars can result from the different orientations of their radio beams with respect to the observer's line of sight. Therefore, determining their emission geometry (e.g., magnetic inclination, viewing angle) is important to gain a better understanding of their properties. However, the lack of knowledge about the phase relationship between the $\gamma$-ray light curves and those in the radio leads to an ambiguity in determining which peak in the $\gamma$-ray light curves is leading (see Trepl et al. 2010 for a more detailed discussion). Therefore, pulsation searches in other wavelengths, in particular in the X-ray, are needed.

So far, only four previous pulsation searches of radio-quiet quasars have been able to detect X-ray pulsation (Halpern \& Holt 1992; Lin et al. 2010, 2013; Marelli et al. 2014). Those pulsars whose periodicities can only be detected in both the $\mathrm{X}$-ray and $\gamma$-ray bands are usually classified as Geminga-like pulsars, with Geminga (i.e., PSR B0633+17) as the prototypical example of this class (Halpern \& Holt 1992; Bertsch et al. 1992). X-ray pulsations of three other known Gemingalike pulsars, PSR J0007+7302 (Lin et al. 2010; Caraveo et al.
2010), PSR J2021+4026 (Lin et al. 2013), and PSR J1813-1246 (Marelli et al. 2014) have been subsequently discovered. Except for PSR J1813-1246, which has a very hard non-thermal dominant X-ray spectrum, the phase-averaged spectrum of the other three Geminga-like pulsars have multi-component spectra that consist of a non-thermal contribution and at least one thermal component (Caraveo et al. 2004; Lin et al. 2010, 2013; Caraveo et al. 2010). The pulse profiles in the X-rays and $\gamma$-rays are dissimilar with an obvious offset (Caraveo et al. 2010; Lin et al. 2010, 2013). This suggests that the pulsed emission in these two regimes originates from different regions. While the aforementioned three pulsars have similar X-ray $/ \gamma$-ray properties, we note that the derived parameters (e.g., characteristic age, magnetic field, and spin-down power) of PSR J0007+7302 and PSR J2021+4026 are rather different from those of Geminga.

On the other hand, the X-ray counterpart of 3EG J1835+5928, RX J1836.2+5925, identified by Mirabal et al. (2000) and Mirabal \& Halpern (2001) has been considered the "next Geminga" for a long time because of the lack of a radio detection (the flux density of the radio observation at a frequency of $1.4 \mathrm{GHz}<3 \mu \mathrm{Jy}$; Abdo et al. 2010), and a spectral behavior and $\gamma$-ray luminosity similar to that of Geminga (Halpern et al. 2002). The $\gamma$-ray pulsation of this source was finally detected by Abdo et al. (2009), and it was classified as a middle-aged pulsar $(\sim 1800 \mathrm{kyr})$ that is similar to Geminga. The surface magnetic field $\left(\sim 5 \times 10^{11} \mathrm{G}\right)$ and the spin-down power $\left(\sim 1.2 \times 10^{34} \mathrm{erg} \mathrm{s}^{-1}\right)$ of PSR J1836+5925 is about one-third of those of Geminga although its spin efficiency in converting $\gamma$-rays is about one order of magnitude higher (Abdo et al. 2010). However, the lack of a pulsation detected in other wavelengths does not allow us to compare the emission properties at different energies. Therefore, periodicity searches at other energies, particularly in the X-ray band, are required to constrain its high energy emission properties and enable us to compare its nature with other Geminga-like pulsars. The X-ray pulsation has been long sought for this target (Halpern et al. 2007; Abdo et al. 2010). However, no positive detection has yet been reported. 
Using the archival XMM-Newton data of PSR J1836+5925, we have performed detailed temporal and spectral analysis observations. In this Letter, we report the results from this investigation. In particular, we report the discovery of X-ray pulsations from PSR J1836+5925 for the first time.

\section{OBSERVATIONS AND DATA ANALYSIS}

The latest XMM-Newton observations of PSR J1836+5925 were on 2013 February 14 and 16 (hereafter 0214 and 0216) and account for total exposure times of of $\sim 44$ and $39 \mathrm{ks}$ (Obs. ID: 0693090101 and 0693090201; PI: G. Pavlov). During these observations, the satellite was pointed to R.A. $=18^{\mathrm{h}} 36^{\mathrm{m}} 13^{\mathrm{s}} .75$, decl. $=+59^{\circ} 25^{\prime} 30^{\prime} 3$ (J2000), which is the timing position of the $\gamma$-ray ephemeris determined by Abdo et al. (2010). In both observations, MOS1/2 CCDs were operated in full-window mode and PN CCD was operated in small-window mode with a temporal resolution of $\sim 5.7 \mathrm{~ms}$ which enables us to search for $\mathrm{X}$-ray pulsations. We reprocessed all the raw data with the tasks emchain and epchain in the XMM-Newton Science Analysis Software (XMMSAS version 13.5.0). For a rigorous analysis, we have excluded those events next to the edges of CCDs and bad pixels, which may have incorrect energies. The good events with "PATTERN" for MOS $1 / 2$ were selected in the range of 0-12 to include single- to quadruple-pixel events, and those for a PN in the range $0-4$ to include only single and double events. In our analysis, we only consider the events in the energy range of $0.2-12 \mathrm{keV}$. We also noted that all investigations have been contaminated by the X-ray background flare. After removing all events that are potentially contaminated, the effective exposures were found to be $41.1 \mathrm{ks}, 41.3 \mathrm{ks}$, and $41.4 \mathrm{ks}$ for MOS1, MOS2, and PN observed on 0214 and $32.3 \mathrm{ks}, 32.2 \mathrm{ks}$, and $32.8 \mathrm{ks}$ for MOS1, MOS2, and PN observed on 0216, respectively. We determined the position of PSR J1836+5925 in each data set with the XMMSAS task edetect_chain.

\subsection{Timing Analysis}

To search for X-ray pulsations from PSR J1836+5925, we utilized the PN data obtained in both observations. The nominal X-ray positions of PSR J1836+5925 on 0214 and 0216 are R.A. $=18^{\mathrm{h}} 36^{\mathrm{m}} 13^{\mathrm{s}} \cdot 68$, decl. $=+59^{\circ} 25^{\prime} 30^{\prime} \cdot 72(\mathrm{~J} 2000)$ (with an uncertainty of $\sim 0^{\prime \prime} 5$ ) and R.A. $=18^{\mathrm{h}} 36^{\mathrm{m}} 13^{\mathrm{s}} .92$, decl. $=+59^{\circ} 25^{\prime} 29^{\prime}$. 64 (with an uncertainty of $\sim 0^{\prime} 7$ ). Within the statistical uncertainties, they are consistent to the position determined by the Chandra/Advanced CCD Imaging Camera, Chandra/High Resolution Camera (HRC; Halpern et al. 2002, 2007 ), and the $\gamma$-ray timing position determined in Abdo et al. (2010). Since the Chandra/HRC provides the most accurate positional determination, we adopted the position determined by this instrument, R.A. $=18^{\mathrm{h}} 36^{\mathrm{m}} 13^{\mathrm{s}} .674$, decl. $=$ $+59^{\circ} 25^{\prime} 30^{\prime} \cdot 15$ (J2000) for the barycentric correction. Events within a circular region of a $20^{\prime \prime}$ radius centered at this position, which corresponds to an encirclingenergy function of $\sim 76 \%$, were extracted. We have 992 counts on 0214 and 806 counts on 0216 available for the timing analysis. We then corrected the photon arrival times to barycentric dynamical time (TDB) with the aforementioned X-ray position and JPL Solar System ephemeris DE 405 using the XMMSAS task of barycen.

We noted that the $\gamma$-ray timing position of PSR J1836+5925 in the available ephemerides has a small offset from our adopted $\mathrm{X}$-ray position. For consistency, we constructed a new local ephemeris of this pulsar to cover the epoch of these recent $X M M$-Newton investigations (i.e., $\sim$ MJD 56338). We used the

Table 1

Local Ephemeris of PSR J1836+5925 Derived from LAT Data which Brackets the Latest XMM-Newton Observations on MJD 56337.45 and MJD 56339.44

Pulsar name

Valid MJD range

Right ascension (R.A.), $\alpha$

Declination (Decl.), $\delta$

Pulse frequency, $f\left(\mathrm{~s}^{-1}\right)$

First derivative of pulse frequency, $\dot{f}\left(\mathrm{~s}^{-2}\right)$

Second derivative of pulse frequency, $\ddot{f}\left(\mathrm{~s}^{-3}\right)$

Epoch of frequency determination (MJD)

Solar system ephemeris model

Time system

Note. The numbers in parentheses denote errors in the last digit.

Fermi Science Tools v9r27p1 package to perform the data reduction. We obtained Fermi Large Area Telescope (LAT) data in the energy range of $0.1-300 \mathrm{GeV}$ within a circular region of interest with a 0.8 radius from the decided X-ray position of PSR J1836+5925. We used Pass 7 data and selected events in the "Source" class (i.e., event class 2). Only a data span of $\sim 1 \mathrm{yr}$, which brackets the epoch of two recent XMM-Newton observations, was considered to avoid accumulated effects due to the timing noise propagated from long-term data. We also excluded events with zenith angles larger than $100^{\circ}$ to reduce contamination from $\gamma$-rays in Earth's albedo. To determine the pulse times of arrival (TOAs), we built a template with the latest ephemeris reported by the Fermi collaboration ${ }^{7}$ by means of Gaussian kernal density estimation (KDE). We have obtained 25 TOAs by cross-correlating the template with the unbinned geocentered data. Each event was assigned to a phase according to the KDE model. With the position fixed at the one determined by the Chandra/HRC (Halpern et al. 2007), we fitted the TOAs with TEMPO2 (Hobbs et al. 2006; Edwards et al. 2006) to obtain a timing solution for PSR J1836+5925 that includes a spin frequency $(f)$, a spin-down rate $(\dot{f})$, and a second derivative of frequency $(f)$. The results are summarized in Table 1 .

Using the $\gamma$-ray ephemeris, which is contemporaneous with recent X-ray observations, we can compare temporal properties in both regimes. We directly folded up the PN data with the spin parameters in Table 1. For the data in $0.2-12 \mathrm{keV}$, the random probability of yielding an X-ray pulsation of 5.7715448470(3) $\mathrm{Hz}$ at an epoch of MJD 56338 is $\sim 3.6 \times 10^{-7}(H \mathrm{TS}=37.1)$ with a single harmonic (de Jager \& Büsching 2010). We have also computed the periodogram of the Rayleigh test in the frequency range $5.76-5.78 \mathrm{~Hz}$ which is shown in Figure 1. It clearly shows an X-ray periodic signal at $5.771545(5) \mathrm{Hz}$ with $Z_{1}^{2}=H \mathrm{TS}=39.5$. The quoted error in the frequency corresponds to the Fourier resolution of this observation (Leahy 1987). This is consistent with the pulse frequency in the $\gamma$-ray regime. To compare the pulse profiles between X-rays and $\gamma$-rays, we folded all the photons extracted from XMM-Newton observations and those obtained from Fermi observations within MJD 56150-56520 in accordance with the $\gamma$-ray ephemeris in Table 1. We also divided the X-ray events obtained into three different energy bands as in the studies of three other Geminga-like pulsars (Caraveo et al. 2004, 2010; Lin et al. 2013). The phase-aligned folded light curves at different energy ranges are shown in Figure 2. For the X-ray light curves, we have subtracted the background sampled from a nearby circular

\footnotetext{
7 https://confluence.slac.stanford.edu/display/GLAMCOG/LAT+Gammaray+Pulsar+Timing+Models
} 


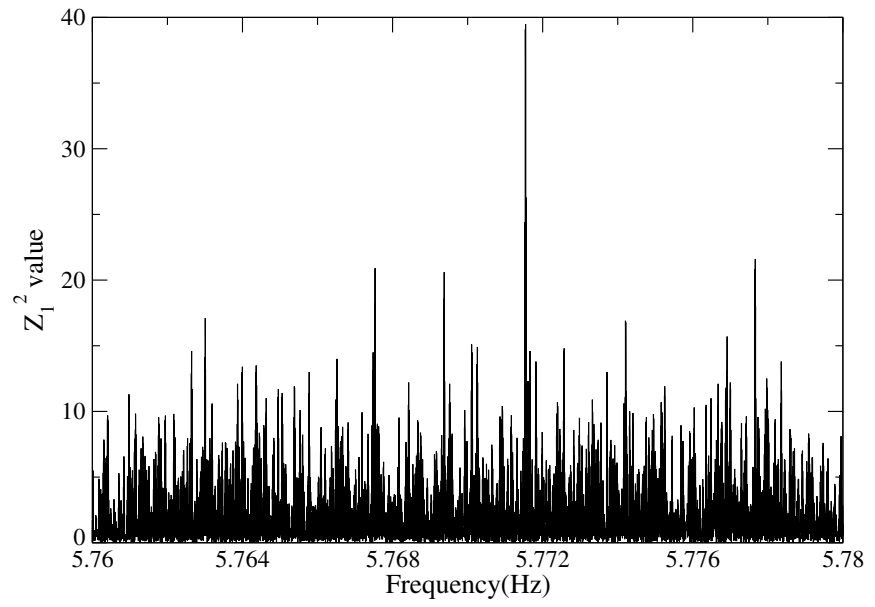

Figure 1. Detection of X-ray pulsation on PSR J1836+5925. The periodogram of the Rayleigh test in the frequency range 5.76-5.78 Hz. An independent trial in the search corresponds to the Fourier resolution of this observation (i.e., $\sim 4.76 \times 10^{-6} \mathrm{~Hz}$ ).

region with a radius of $20^{\prime \prime}$ centered at R.A. $=18^{\mathrm{h}} 36^{\mathrm{m}} 20^{\mathrm{s}} .752$, decl. $=+59^{\circ} 26^{\prime} 07^{\prime \prime} .92(\mathrm{~J} 2000)$. The X-ray pulsation can be firmly detected in the soft X-ray band $(H$ TS $=23.6$ with a random probability of $\sim 7.9 \times 10^{-5}$ ) and marginally found in the medium X-ray band $(H$ TS $=11.6$ with a random probability of $\left.\sim 9.7 \times 10^{-3}\right)$. Nevertheless, the significance is too low to claim detection in the hard band $(H \mathrm{TS}=4.0$ with a random probability of 0.2 ), which can be ascribed to the small photon statistic.

\subsection{Spectral Analysis}

Because our adopted data have total exposures of more than $80 \mathrm{ks}$, this enables us to place a tighter constraint on the X-ray spectral properties of PSR J1836+5925 compared with previous investigations (Halpern et al. 2002; Abdo et al. 2010). We extracted the spectra of PSR J1836+5925 from a circle with a radius of $20^{\prime \prime}$ centered at its nominal position in each set of EPIC data. The background spectra were sampled from a nearby circular source-free region with a radius of $20^{\prime \prime}$ in each individual data set. We generated the response matrices and ancillary response files with the XMMSAS tasks rmfgen and arfgen. Since the X-ray emission from PSR J1836+5925 is relatively soft, we considered X-ray spectral fits in the energy range of $0.2-5 \mathrm{keV}$. We grouped each spectrum so as to have a minimum of 15 counts in each bin. We have examined each data set individually and found that the inferred parameters are consistent within the uncertainties. In order to optimize the photon statistics, we fitted all the EPIC data obtained in these two observations simultaneously. The errors in the spectral parameters quoted in this Letter are in $1 \sigma$ for two parameters of interest (i.e., $\Delta \chi^{2}=2.30$ above the minimum) for singlecomponent models and in $1 \sigma$ for four parameters of interest (i.e., $\Delta \chi^{2}=4.72$ above the minimum) for multi-component models.

For the single-component models tested (i.e., power law and blackbody), none of them resulted in a statistically reasonable fit (with a reduced $\chi^{2}>1.5$ ). This may indicate that the $\mathrm{X}$ ray emission of PSR J1836+5925 comprises more than one spectral component. Therefore, we proceeded to fit the data with multi-component spectral models. We found that the spectrum can be described by an absorbed blackbody plus power-law model. We first attempted to perform the fitting

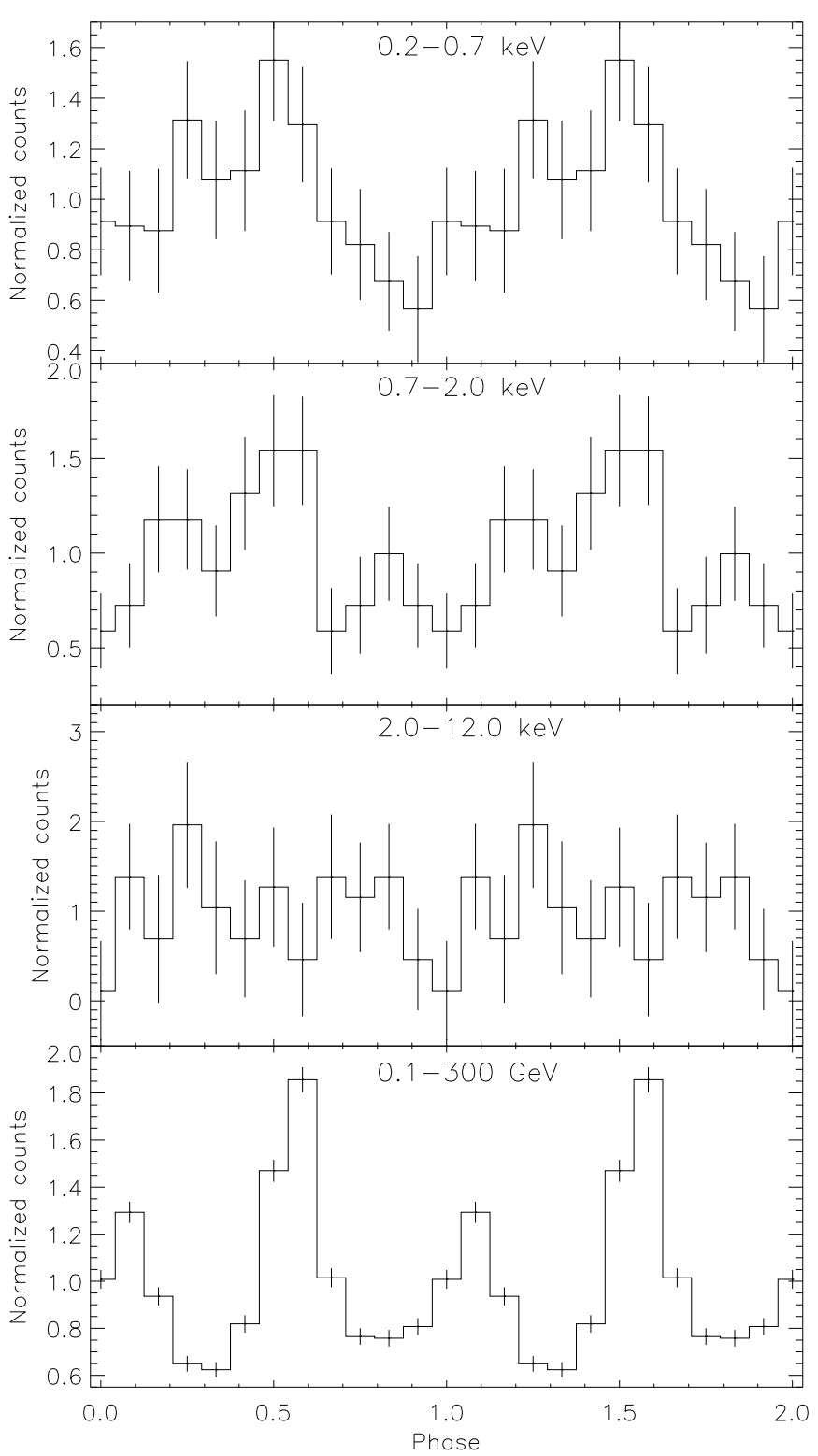

Figure 2. Folded light curves of PSR J1836+5925 in different energy ranges. Each panel shows the pulse profile of 12 bins in the energy band specified in the legend. The epoch/phase zero of all the profiles were determined at MJD 56338 shown in Table 1.

with all parameters free, which yields a column absorption of $n_{\mathrm{H}}<8.5 \times 10^{19} \mathrm{~cm}^{-2}$, a photon index of $\Gamma=1.8 \pm 0.3$, a powerlaw model normalization of $6.0_{-1.2}^{+1.3} \times 10^{-6}$ photons $\mathrm{keV}^{-1} \mathrm{~cm}^{-2}$ $\mathrm{s}^{-1}$ at $1 \mathrm{keV}$, and a blackbody temperature of $k T=62_{-10}^{+11} \mathrm{eV}$ with an emission radius of $R=1.32_{-0.55}^{+2.37} \mathrm{~km}$ at $800 \mathrm{pc}$. This resulted in a desirable goodness-of-fit $\left(\chi^{2}=90.46\right.$ for 85 dof $)$.

As the column absorption cannot be properly constrained, we also fitted the data with $n_{\mathrm{H}}$ fixed at a total Galactic H I column density of $4 \times 10^{20} \mathrm{~cm}^{-2}$ in the direction of PSR J1836+5925 (Kalberla et al. 2005). This yielded the best-fit parameters of $\Gamma=2.1 \pm 0.3$, a power-law model normalization of $(7.1 \pm 1.4) \times 10^{-6}$ photons $\mathrm{keV}^{-1} \mathrm{~cm}^{-2} \mathrm{~s}^{-1}$ at $1 \mathrm{keV}$, $k T=45_{-8}^{+10} \mathrm{eV}$, and a blackbody radius of $R=6.3_{-3.4}^{+7.8} \mathrm{~km}$. The corresponding goodness-of-fit is $\chi^{2}=101.8$ for 86 dof which is statistically acceptable. The best-fit model and the observed spectra are shown in Figure 3. 


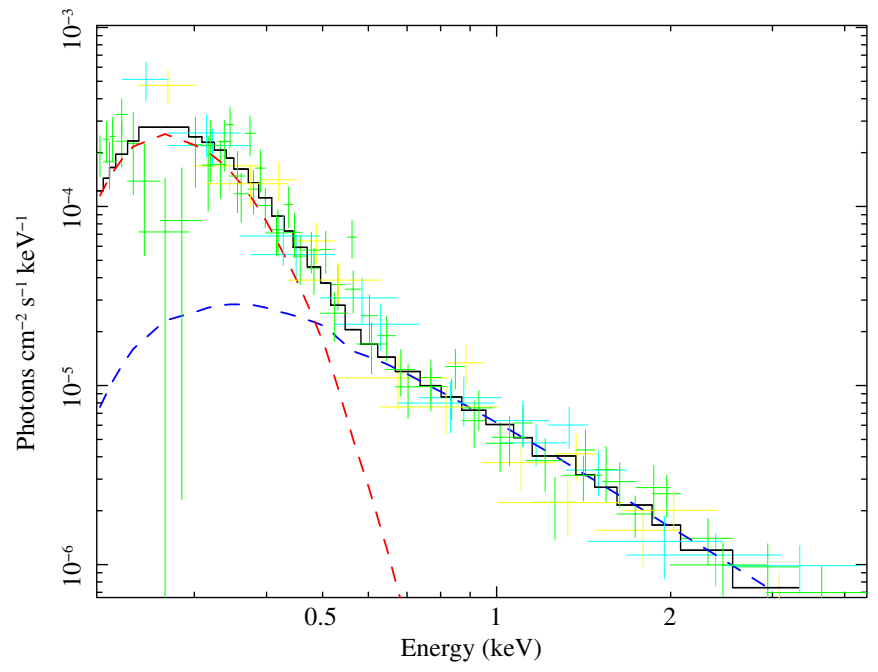

Figure 3. Phase-averaged X-ray spectra of PSR J1836+5925 as observed by XMM-Newton (MOS 1: cyan; MOS 2: yellow; PN: green) and simultaneously fitted to an absorbed power law plus blackbody model. The best-fit model for the thermal (red dashed line) and non-thermal (blue dashed line) components are illustrated separately.

(A color version of this figure is available in the online journal.)

We have also considered the spectral fit with a dual blackbody model. However, the goodness-of-fit yielded $\left(\chi^{2}=109.3\right.$ for 85 dof) by this model is worse than the power law plus blackbody model. Fixing the $n_{\mathrm{H}}$ at the Galactic H I value does not result in any improvement in the fitting.

\section{DISCUSSION}

In this Letter, we report the discovery of X-ray pulsations from PSR J1836+5925. We found that the pulsed events contribute in both the soft and medium bands $(0.2-2 \mathrm{keV})$. On the other hand, no indication of pulsation in the hard band $(>2 \mathrm{keV})$ has been found in our investigation. The X-ray and $\gamma$-ray pulsation from PSR J1836+5925 have significantly different behavior. The pulsed $\gamma$-rays have a structure of relatively sharp double peaks that presumably originate from the curvature radiation emitted from the pair creation region in the magnetosphere. The spin period of $P=173 \mathrm{~ms}$ and the characteristic age of $\tau=1.8$ Myr suggest PSR J1836+5925 as a mature pulsar. Assuming the emission is isotropic, the $\mathrm{GeV} \gamma$-ray efficiency of PSR J1836+5925 is $\eta=\left(L_{\gamma} / \dot{E}\right) \times 100 \%=180 \% \pm 1_{-100}^{+200} \%$ (second Fermi pulsar catalog; Abdo et al. 2013), where $\dot{E}$ is the spin down power. The first uncertainty in $\eta$ comes from the statistical uncertainties in the spectral fit and the second is due to the distance uncertainty (Abdo et al. 2013). This high efficiency in $\mathrm{GeV}$ emission is similar to $\eta=97.4 \% \pm 0.3_{-50}^{+300} \%$ of a typical mature pulsar, Geminga, if one assumes a $4 \pi$ solid angle. Considering the possible overestimated distances and/or the beaming factor, the aforementioned conversion efficiencies can be overestimated. Taking the more realistic parameters into account, this might result in an efficiency $\eta<100 \%$.

In order to compare with Geminga, therefore, we discuss the origin of the X-ray emission of PSR J1836+5925. In the soft band (see Figure 2), the light curve is essentially sinusoidal which suggests the X-ray pulsation mostly originates from the modulation of the heated polar cap. This is consistent with the presence of a blackbody component inferred from the spectral fit.
To account for the power-law component, we discuss it in the context of the outer gap model (Cheng \& Zhang 1998). According to this model, the non-thermal component of a canonical pulsar is synchrotron emission from the relativistic $e^{-} / e^{+}$in the outer magnetospheric gap (Takata et al. 2006, 2008) with a weak absorption. The photon index is expected to be in the range 1.5-2.0 which is consistent with the best-fit value. For the light curve in $0.7-2 \mathrm{keV}$, its profile is apparently different from that in $0.2-0.7 \mathrm{keV}$ which might suggest a different origin. In examining the relative flux contributions from the thermal and non-thermal component, we found that while the nonthermal flux takes $\sim 15 \%$ in $0.2-0.7 \mathrm{keV}$ its contribution is $>99 \%$ in $0.7-2 \mathrm{keV}$ (see also Figure 3). This suggests that $\mathrm{X}$-ray pulsations seen in $0.2-0.7 \mathrm{keV}$ and $0.7-2 \mathrm{keV}$ might have a different origin. However, the small photon statistics in the medium band preclude any firm conclusion. To better characterize the X-rays from PSR J1836+5925 in different energy bands, a deeper observation is certainly required. With a larger number of photons detected, we can also perform a phaseresolved spectroscopy to investigate how the spectral properties vary across rotational phase, which would provide important input for a physical modeling of the pulsar.

We thank Mr. Jason H. K. Wu of the Max-Planck-Institut für Radioastronomie for providing useful comments on the temporal analysis techniques. This work made use of data supplied by the High Energy Astrophysics Science Archive Research Center (HEASARC). This project is partially supported by the Ministry of Science and Technology of Taiwan through grant NSC 101-2112-M-039-001-MY3. C.Y.H. is supported by the National Research Foundation of Korea through grant 2011002383. J.T. is supported by a GRF grant from the Hong Kong Government under HKU700911P. C.P.H. and Y.C. are supported by the Ministry of Science and Technology of Taiwan through grant NSC 102-2112-M-008-020-MY3. A.K.H.K. is supported by the Ministry of Science and Technology of Taiwan through grants NSC 100-2628-M-007-002-MY3 and NSC 100-2923M-007-001-MY3. D.C.C.Y. receives financial support from the Ministry of Science and Technology of Taiwan through grant NSC 102-2115-M-030-003 and another FJU project, A0502004.

\section{REFERENCES}

Abdo, A. A., Ackermann, M., Ajello, M., et al. 2009, Sci, 325, 840 Abdo, A. A., Ackermann, M., Ajello, M., et al. 2010, ApJ, 712, 1209 Abdo, A. A., Ajello, M., Allafort, A., et al. 2013, ApJS, 208, 17

Bertsch, D. L., Brazier, K. T. S., Fichtel, C. E., et al. 1992, Natur, 357, 306 Caraveo, P. A., De Luca, A., Marelli, M., et al. 2010, ApJL, 725, L6

Caraveo, P. A., De Luca, A., Mereghetti, S., Pellizzoni, A., \& Bignami, G. F. 2004, Sci, 305, 376

Cheng, K. S., \& Zhang, L. 1998, ApJ, 498, 327

de Jager, O. C., \& Büsching, I. 2010, A\&A, 517, L9

Edwards, R. T., Hobbs, G. B., \& Manchester, R. N. 2006, MNRAS, 372, 1549

Halpern, J. P., Camilo, F., \& Gotthelf, E. V. 2007, ApJ, 668, 1154

Halpern, J. P., Gotthelf, E. V., Mirabal, N., \& Camilo, F. 2002, ApJL, 573, L41

Halpern, J. P., \& Holt, S. S. 1992, Natur, 357, 222

Hobbs, G. B., Edwards, R. T., \& Manchester, R. N. 2006, MNRAS, 369, 655 Kalberla, P. M. W., Burton, W. B., Hartmann, D., et al. 2005, A\&A, 440, 775 Kerr, M. 2011, ApJ, 732, 38

Leahy, D. A. 1987, A\&A, 180, 275

Lin, L. C. C., Huang, R. H. H., Takata, J., et al. 2010, ApJL, 725, L1

Lin, L. C. C., Hui, C. Y., Hu, C. P., et al. 2013, ApJL, 770, L9

Marelli, M., Harding, A., Pizzocaro, D., et al. 2014, arXiv:1407.1748

Mirabal, N., \& Halpern, J. P. 2001, ApJL, 547, L137

Mirabal, N., Halpern, J. P., Eracleous, M., \& Becker, R. H. 2000, ApJ, 541, 180

Takata, J., Chang, H., \& Shibata, S. 2008, MNRAS, 386, 748

Takata, J., Shibata, S., Hirotani, K., \& Chang, H.-K. 2006, MNRAS, 366, 1310

Trepl, L., Hui, C. Y., Cheng, K. S., et al. 2010, MNRAS, 405, 1339 